\title{
Properties of the Bound States on a Hexagonal Lattice
}

\author{
M. BAK \\ Institute of Physics, A. Mickiewicz University \\ Umultowska 85, 61-614 Poznań, Poland
}

\begin{abstract}
Two electrons on a lattice pose an interesting problem which can be solved exactly. When the effective interactions are strong enough, the bound states are formed. The properties of such bound pairs in an extended Hubbard model are examined for both signs of nearest neighbor hopping integral $t$. The stabilization of $s^{*}$-wave pairing is found for $t<0$ and of $f$-wave for $t>0$. Phase diagrams for the existence of none, one or two $s^{*}$-wave solutions are calculated.
\end{abstract}

PACS numbers: 71.10.Fd, 74.20.Rp, 71.27.+a, 71.10.Hf

\section{Introduction}

Since the discovery of high temperature superconductivity (HTS) the theoretical effort to understand its properties has not stopped. Despite a lot of work, there is consensus about few things. In particular, there are very few exact results. One of them is a solution of the bound state of two-electron pair on a lattice. The connection with the superconductivity is by a theorem of Randeria et al. [1], saying that the critical value of the nearest-neighbor (n.n.) interactions for appearing of the bound, two-electron state is also a critical value for appearing of the superconductivity of the $s$-wave symmetry in two dimensions $(d=2)$.

On the other hand, the two-electron problem is low density (and also strong-coupling) limit of BCS equations. Thus it may be relevant to the phenomenon of the pseudogap as well as to the BCS-BEC (Bose-Einstein condensation) crossover, in particular to the problem of the Bose-Einstein condensation of pre-formed, bound pairs, thought to be one of the possible mechanisms of HTS.

The solution of two-electron bound states, treated as a limiting case of low-density paired electron systems may shed light not only on HTS but also on phenomena in other strongly correlated materials like heavy fermion systems or cobalt-based superconductors [2].

While the properties of two-electron states on a square lattice were partly examined [3-9] (the general properties of bound (magnon) states given by Mat- 
tis [10]) the hexagonal lattice possessed by, e.g., cobaltates or many heavy electron systems, is much less explored [11]. The present paper tries to close this gap.

\section{The formalism}

The model of interest is an extended Hubbard model on a hexagonal lattice

$$
H=t \sum_{\langle i j\rangle \sigma}\left(c_{i \sigma}^{\dagger} c_{j \sigma}+\text { h.c. }\right)+U \sum_{i} n_{i \uparrow} n_{i \downarrow}+W \sum_{\langle i j\rangle} \sum_{\sigma \sigma^{\prime}} n_{i \sigma} n_{j \sigma^{\prime}},
$$

where $t$ is the hopping integral, $\langle i j\rangle$ means n.n., $U$ is on-site and $W$ is inter-site (between n.n.) effective interaction. Numerical results in this paper are obtained for negative (attractive) values of $U$ and $W$. Thus the present paper will extend and generalize the results of Ref. [11], which were obtained for $U>0$.

One of possible causes of attraction between charges is, e.g., interaction via phonons like in BCS theory. Other possible sources of parameter renormalization are interactions with other (quasi)bosonic degrees of freedom like excitons or plasmons, coupling of electrons to other electron subsystems in the solid or other purely chemical mechanisms [3]. Let us also note that while the n.n. $W$-term has its contributions in the singlet and triplet pairing channels with the same sign, the Heisenberg term, $J \boldsymbol{S}_{i} \cdot \boldsymbol{S}_{j}$, added to the Hamiltonian, yields the contribution in both channels with the opposite sign. Thus we may obtain the effective attractive pairing in one of the channels even for all the Hamiltonian parameters being positive.

We will also consider both the case of positive and negative $t$. Because the lattice is not bipartite, these two cases are not equivalent. The band energy in the lattice of interest is given by the formula

$$
\varepsilon_{k}=2 t \cos \left(k_{x}\right)+4 t \cos \left(\frac{k_{x}}{2}\right) \cos \left(\frac{\sqrt{3}}{2} k_{y}\right) .
$$

We expand the wave function in the basis of the two electron states given by the plane waves, with definite quasimomenta. Such states are the eigenstates of the hopping part of the Hamiltonian Eq. (1). We will write them down in the center of mass coordinate system as $|\boldsymbol{P}, \boldsymbol{q}\rangle$, where $\boldsymbol{P}$ is the center of mass momentum and $\boldsymbol{q}$ is the relative momentum. The wave function is a sum of all possible such states with appropriate weights $F_{\boldsymbol{P}}(\boldsymbol{q})$ :

$$
\Psi_{\boldsymbol{P}}=\sum_{\boldsymbol{q}} F_{\boldsymbol{P}}(\boldsymbol{q})|\boldsymbol{P}, \boldsymbol{q}\rangle
$$

The set of coefficients $F_{\boldsymbol{P}}(\boldsymbol{q})$ fully describes the system. By the action of the Hamiltonian in $\boldsymbol{k}$-space, we can obtain in a standard way the equation of motion for $F_{\boldsymbol{P}}(\boldsymbol{q})$ :

$$
\begin{aligned}
(E- & \left.\varepsilon_{\boldsymbol{P} / 2-\boldsymbol{q}}-\varepsilon_{\boldsymbol{P} / 2+\boldsymbol{q}}\right) F_{\boldsymbol{P}}(\boldsymbol{q}) \\
& =U \sum_{\boldsymbol{k}} F_{\boldsymbol{P}}(\boldsymbol{k})+W \sum_{\delta} \mathrm{e}^{-\mathrm{i} \boldsymbol{q} \delta} \sum_{\boldsymbol{k}} F_{\boldsymbol{P}}(\boldsymbol{k}) \mathrm{e}^{-\mathrm{i} \boldsymbol{k} \delta} .
\end{aligned}
$$


By recalling that the real space wave function is the Fourier transform: $\Psi_{\boldsymbol{P}}(\boldsymbol{r})=$ $(1 / N) \sum_{\boldsymbol{q}} F_{\boldsymbol{P}}(\boldsymbol{q}) \exp (-\mathrm{i} \boldsymbol{q} \cdot \boldsymbol{r})$, we can obtain

$$
\Psi_{\boldsymbol{P}}(\boldsymbol{r})=\sum_{\boldsymbol{r}^{\prime}} G\left(E, \boldsymbol{P}, \boldsymbol{r}, \boldsymbol{r}^{\prime}\right) V\left(\boldsymbol{r}^{\prime}\right) \Psi_{\boldsymbol{P}}\left(\boldsymbol{r}^{\prime}\right)
$$

where

$$
G\left(E, \boldsymbol{P}, \boldsymbol{r}, \boldsymbol{r}^{\prime}\right)=\frac{1}{N} \sum_{\boldsymbol{q}} \frac{\mathrm{e}^{-\mathrm{i} \boldsymbol{q}\left(\boldsymbol{r}-\boldsymbol{r}^{\prime}\right)}}{E-\varepsilon_{\boldsymbol{P} / 2-\boldsymbol{q}}-\varepsilon_{\boldsymbol{P} / 2+\boldsymbol{q}}} .
$$

If $\boldsymbol{r}$ belongs to a set describing the position of an atom in the center of coordinate system and its n.n., then Eq. (5) becomes a matrix equation

$$
\check{\Psi}=\check{G}(E, \boldsymbol{P}) \check{V} \check{\Psi}
$$

where $\check{\Psi}$ is a vector, whose components are the wave functions in position space at respective $\boldsymbol{r}$ 's. In the notation, where the first element has an index zero, the first element, $\Psi_{0}$, is the wave function at the center of the coordinate system, $\Psi_{1}, \Psi_{2}, \ldots, \Psi_{6}$ are the wave functions at the positions of n.n. (anticlockwise). $\check{V}$ is diagonal interaction matrix, with $V_{00}=U$ and $V_{n n}=W, n=1,2, \ldots, 6$ and elements of $\breve{G}$ are given by Eq. (6), where both $\boldsymbol{r}$ and $\boldsymbol{r}^{\prime}$ belong to a set $r_{0}, r_{1}, \ldots, r_{6}$.

By a similarity transformation we can cast vector $\Psi$ into the form, such that its components are the eigenfunctions of the symmetry operations of the point group of the lattice (for hexagonal lattice it is $C_{6 v}$ ). The transformation matrix can be easily read off from the character table of the symmetry group and is given below

$$
\left(\begin{array}{ccccccc}
1 & 0 & 0 & 0 & 0 & 0 & 0 \\
0 & 1 / \sqrt{6} & 1 / \sqrt{6} & 1 / \sqrt{6} & 1 / \sqrt{6} & 1 / \sqrt{6} & 1 / \sqrt{6} \\
0 & 2 / \sqrt{12} & -1 / \sqrt{12} & -1 / \sqrt{12} & 2 / \sqrt{12} & -1 / \sqrt{12} & -1 / \sqrt{12} \\
0 & \mathrm{i} / \sqrt{6} & -\mathrm{i} / \sqrt{6} & \mathrm{i} / \sqrt{6} & -\mathrm{i} / \sqrt{6} & \mathrm{i} / \sqrt{6} & -\mathrm{i} / \sqrt{6} \\
0 & 2 \mathrm{i} / \sqrt{12} & \mathrm{i} / \sqrt{12} & -\mathrm{i} / \sqrt{12} & -2 \mathrm{i} / \sqrt{12} & -\mathrm{i} / \sqrt{12} & \mathrm{i} / \sqrt{12} \\
0 & 0 & -1 / 2 & 1 / 2 & 0 & -1 / 2 & 1 / 2 \\
0 & 0 & \mathrm{i} / 2 & \mathrm{i} / 2 & 0 & -\mathrm{i} / 2 & -\mathrm{i} / 2
\end{array}\right)
$$

After the transformation, in the numerator of the Green function of Eq. (6) there will appear the combinations of exponents, transforming according to the irreducible representations (irreps) of the symmetry group of the lattice. The symbols of those irreps will be the new indexes of the Green function in the new form

$$
G_{m n}(\boldsymbol{P}, E)=\frac{1}{N} \sum_{\boldsymbol{q}} \frac{\phi_{n}(\boldsymbol{q}) \phi_{m}(\boldsymbol{q})}{E-\varepsilon_{\boldsymbol{P} / 2-\boldsymbol{q}}-\varepsilon_{\boldsymbol{P} / 2+\boldsymbol{q}}},
$$

where 


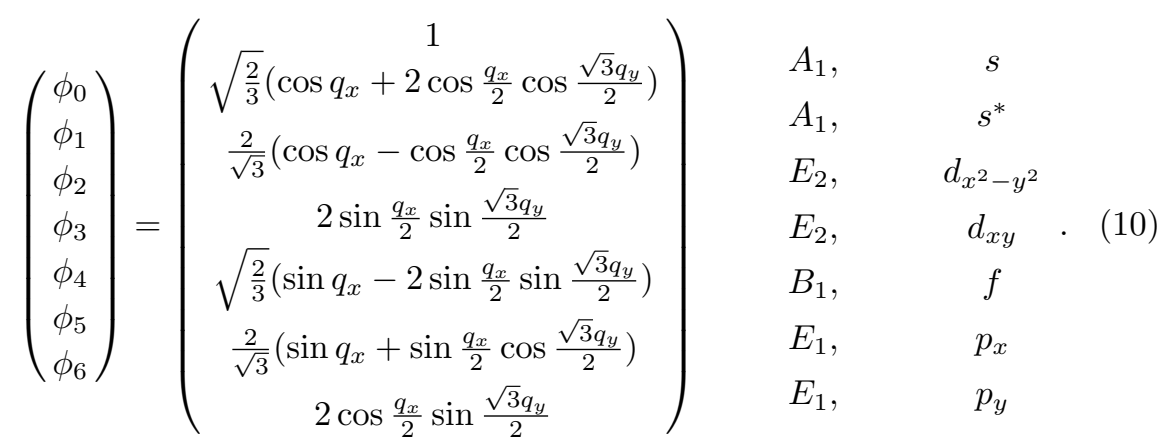

In the two rightmost columns there are, respectively: symbols of the irreps, according to which a given $\phi_{n}$ transforms and symbols traditionally labeling wave functions of given symmetry. In Fig. 1 there is a contour plot of the above functions superimposed on the outline of the Brillouin zone.
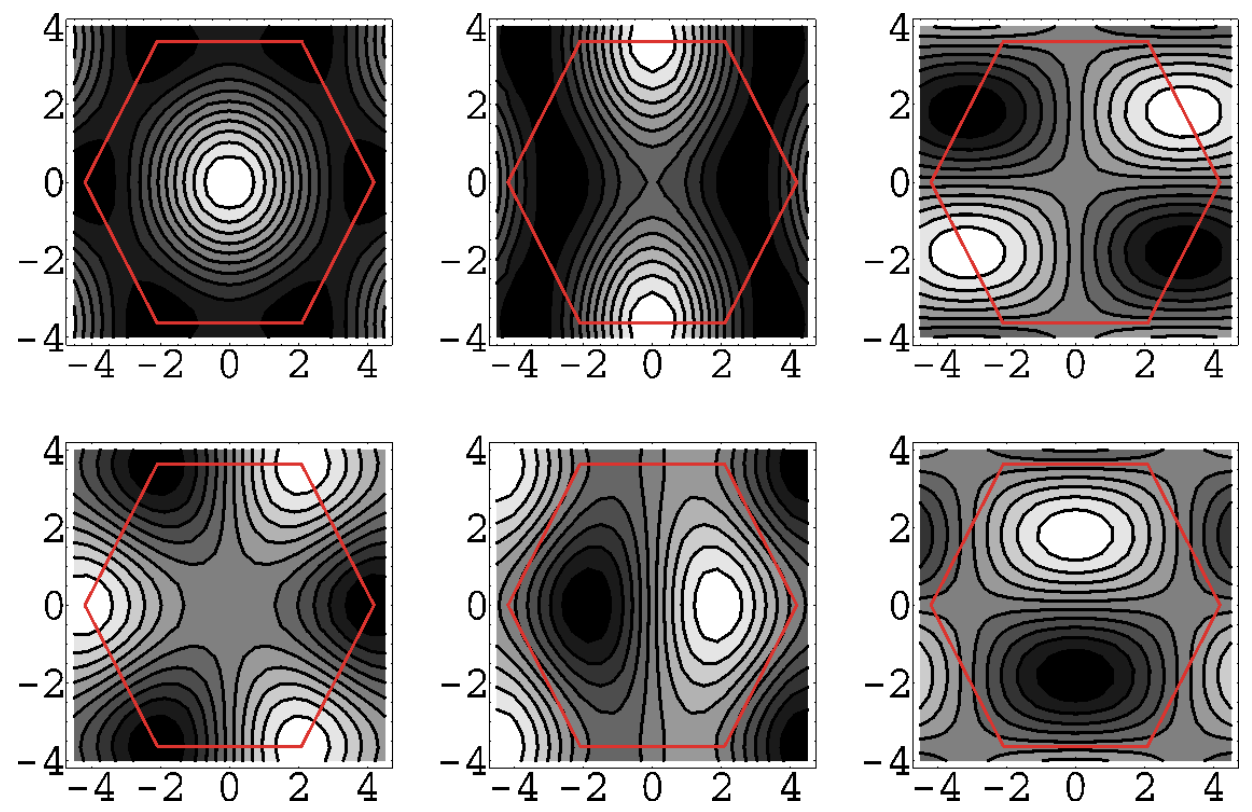

Fig. 1. Outline of the wave functions, which transform according to the irreducible representations of the symmetry group of hexagonal lattice $C_{6 v}$. Top row from the left: $s^{*}, d_{x^{2}-y^{2}}, d_{x y}$, bottom row from the left: $f, p_{x}, p_{y}$, according to the notation given in Eq. (10).

To find eigenenergies we have to solve Eq. (7), which has nontrivial solutions when

$$
\operatorname{det}(1-\check{G} \check{V})=0 \text {. }
$$

Thanks to the described transformation the determinant (Eq. (11)) decouples in the various points of the Brillouin zone (B.z.). In the $\Gamma$ point, only the $s$ and $s^{*}$ 
(extended-s) pairings stay coupled, yielding an equation

$$
\operatorname{det}\left(\begin{array}{cc}
1 / U-G_{00} & -G_{01} \\
-G_{10} & 1 / W-G_{11}
\end{array}\right)=0 .
$$

The other pairings in the $\Gamma$ point are given by

$$
1 / W=G_{n n}, \quad \text { where } n=2,3, \ldots, 6 .
$$

On $\Gamma-K$ line $d_{x y}$ - and $p_{y}$-wave pairings separate completely, while the pairings $s+s^{*}+d_{x^{2}+y^{2}}$ and $p_{x}+f$ stay coupled. In other parts of B.z. the determinant of Eq. (11) decouples only into singlet $s+s^{*}+d_{x^{2}+y^{2}}+d_{x y}$ and triplet $p_{x}+p_{y}+f$ pairings.

During calculation of critical values from Eq. (12) we have to deal with the divergence of $\tilde{G}_{00}, \tilde{G}_{01}, \tilde{G}_{10}$ and $\tilde{G}_{11}$, where $\tilde{G}_{m n}$ means Green function $G_{m n}(E)$ at the band bottom (i.e., $E=-12|t|$ for $t<0$ and $E=-6 t$ for $t>0$ ). The way to circumvent this problem is to divide the Green functions into divergent and non-divergent part and then to make the coefficient at the divergent part equal to zero. The following equalities come in handy

- for $t<0$ :

$$
\begin{aligned}
& \tilde{G}_{11}-6 \tilde{G}_{00}=1 / 2, \\
& \tilde{G}_{01}-\sqrt{6} \tilde{G}_{00}=1 /(2 \sqrt{6}),
\end{aligned}
$$

- for $t>0$ :

$$
\begin{aligned}
& \tilde{G}_{11}-(3 / 2) \tilde{G}_{00}=1 / 4, \\
& \tilde{G}_{01}+\sqrt{3 / 2} \tilde{G}_{00}=-1 /(2 \sqrt{6}) .
\end{aligned}
$$

\section{Results}

In Fig. 2 there are eigenenergy solutions for different pairings across the B.z. for $t<0$. The pairing most strongly stabilized around the minimum of the band energy in the $\Gamma$ point is $s^{*}$-wave. Let us note that we can unambiguously label the pairings in the $\Gamma$ point only. In any other part of the B.z. pure pairings do not exist (except for $p_{y}$ and $d_{x y}$ on the $\Gamma-K$ line), due to equation coupling; we have admixtures of different pairings in different proportions, depending on the position in B.z. In most of B.z. we can just tell the difference between singlet and triplet pairing. In some cases we can label the pairing, tracing curves to the $\Gamma$ point. This way we can identify the lowest state on $K-M$ line as $p$-wave, a fact interesting in itself, as the ground state around the minimum of the band in $\Gamma$ point is $s^{*}$-wave. The weakest bound state due to intersite interactions is triplet $f$-wave (we did not consider pure $s$-wave, which depends only on $U$ ).

To create a bound pair at the bottom of the band $(E /|t|=-12)$ a critical value of $W$ must be reached for $p$-wave pairing $\left(W_{\mathrm{c}} /|t|=-7.092\right), d$-wave pair$\operatorname{ing}\left(W_{\mathrm{c}} /|t|=-12.988\right)$, and $f$-wave pairing $\left(W_{\mathrm{c}} /|t|=-15.623\right)$. The pairs of $s^{*}$-wave type require only infinitesimal on-site attraction (for $W=0$ ) or infinitesimal inter-site attraction (for $U=0$ ). For finite $U$ and $W$ the threshold value must 


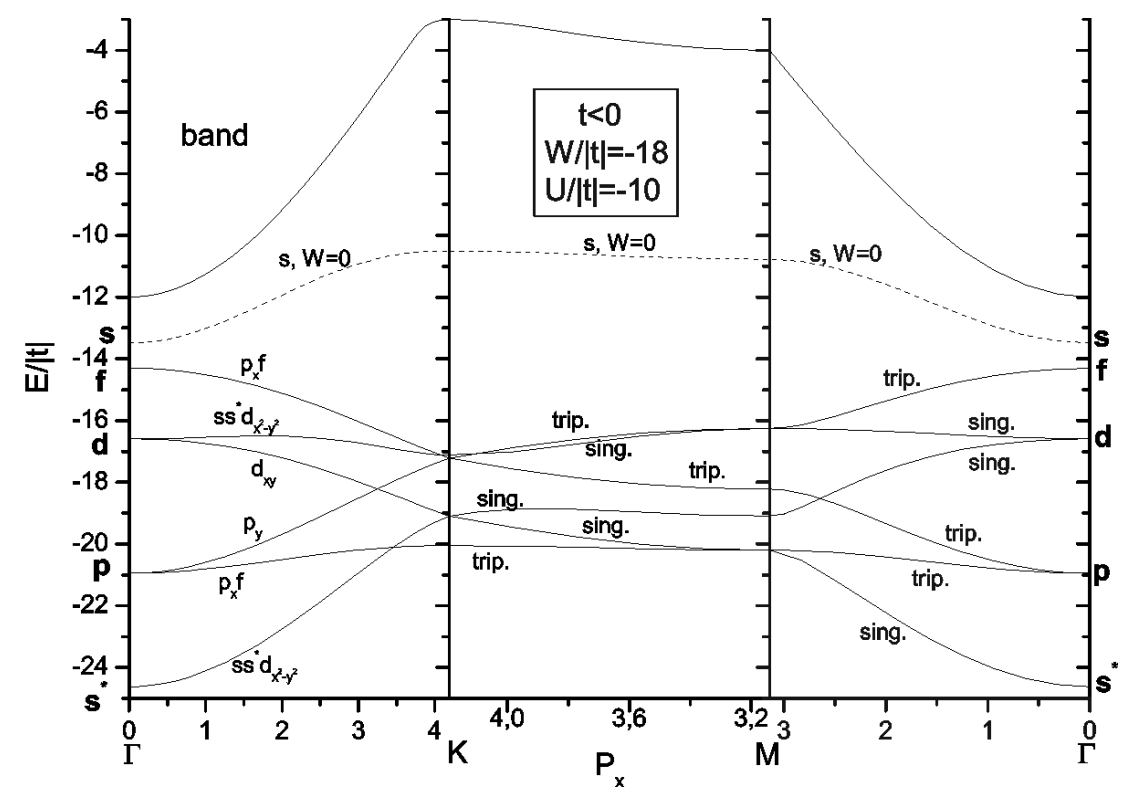

Fig. 2. Dispersion curves across the B.z. for $t<0, W /|t|=-18$ and $U /|t|=-10$. Label "trip." means one of the triplet solutions of the coupled $p_{x}+p_{y}+f$-wave pairings; "sing." denotes one of three or four solutions of the four coupled equations for the singlet $s+s^{*}+d_{x^{2}-y^{2}}+d_{x y}$-wave pairing. Dotted line denotes pure $s$-wave pairing.

be reached, which is a function of these parameters. A critical value for $W$ for $t<0$ is given by the formula

$$
W_{\text {cr }} /|t|=\frac{-2 U}{12|t|+U} .
$$

For infinite on-site repulsion the intersite attraction must reach $W=-2|t|$ to form a bound state. For infinite intersite repulsion the on-site attraction needed for formation of a bound state is $-2|t|$ times the number of n.n., i.e., $U=-12|t|$. The plot of the formula of Eq. (18) is given in Fig. 3. Let us note that in the plot there are two critical lines. There are two possible bound states of $s^{*}$-wave symmetry, with different properties, in particular one of them is much stronger bound than the other.

The binding energy is shown in Fig. 4. We can compare behavior of the solutions from the area with one and with two solutions in Fig. 3. All curves begin, for $E=0$, at their respective critical values. The $s s^{*}$ for $U=0$ curve begins at 0 , as was told before. The curve for $U=-10|t|$ begins for some positive critical value of $W$ and its energy grows with decreasing $W$. It is the only solution of $2 \times 2$ determinant for $s s^{*}$-wave pairing in $\Gamma$ point. For $U=-15|t|$ there are two solutions. The energy of the first one never drops below a finite asymptotic value. The other starts at the pretty large, critical value and also grows with decreasing $W$ but much more slowly than other pairings. 


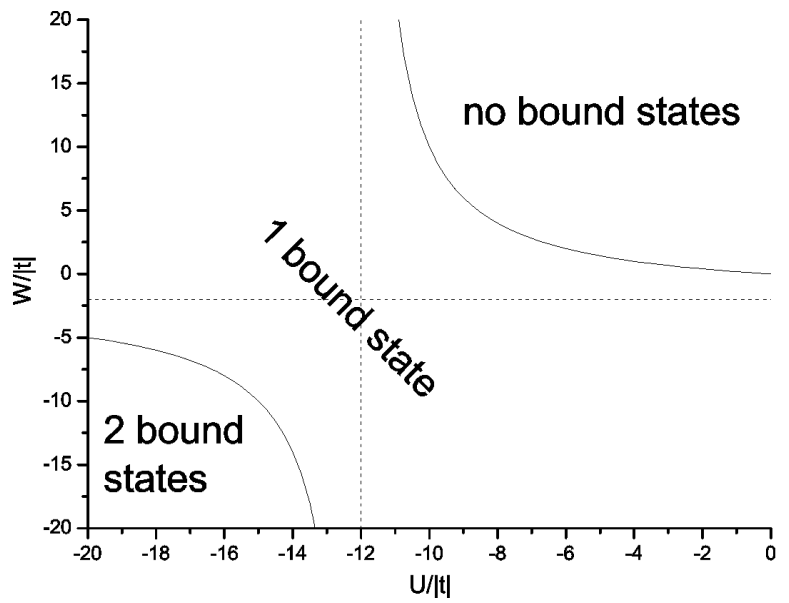

Fig. 3. Critical value for creation of bound pair for $t<0$.

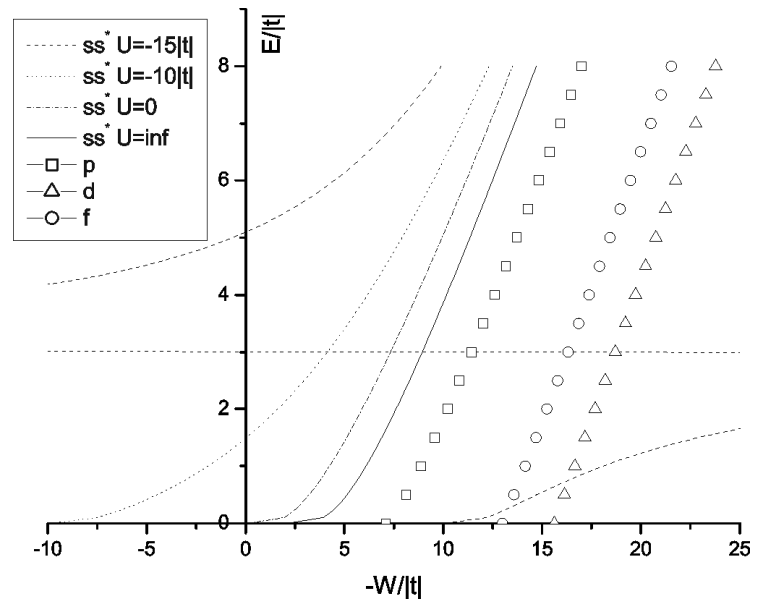

Fig. 4. Binding energy of pairs of different symmetries for $t<0 . U=$ inf means infinite on-site repulsion. Horizontal dashed line is the asymptote for the two branches of the $U=-15|t|$ solution: dashed leftmost and dashed rightmost.

An example of the described behavior could be seen in Fig. 2, where there are only three singlet lines, despite they are calculated from the determinant $4 \times 4$ $(2 \times 2$ in the $\Gamma$ point) and there should be four solutions. For $U=-10|t|$ we are in the area of the phase diagram of Fig. 3, where one of the solutions does not exist.

In Fig. 5, for $t>0$, there are four singlet lines, as the critical value in this case is given by the formula

$$
W_{\mathrm{cr}} / t=\frac{-4 U}{6 t+U}
$$




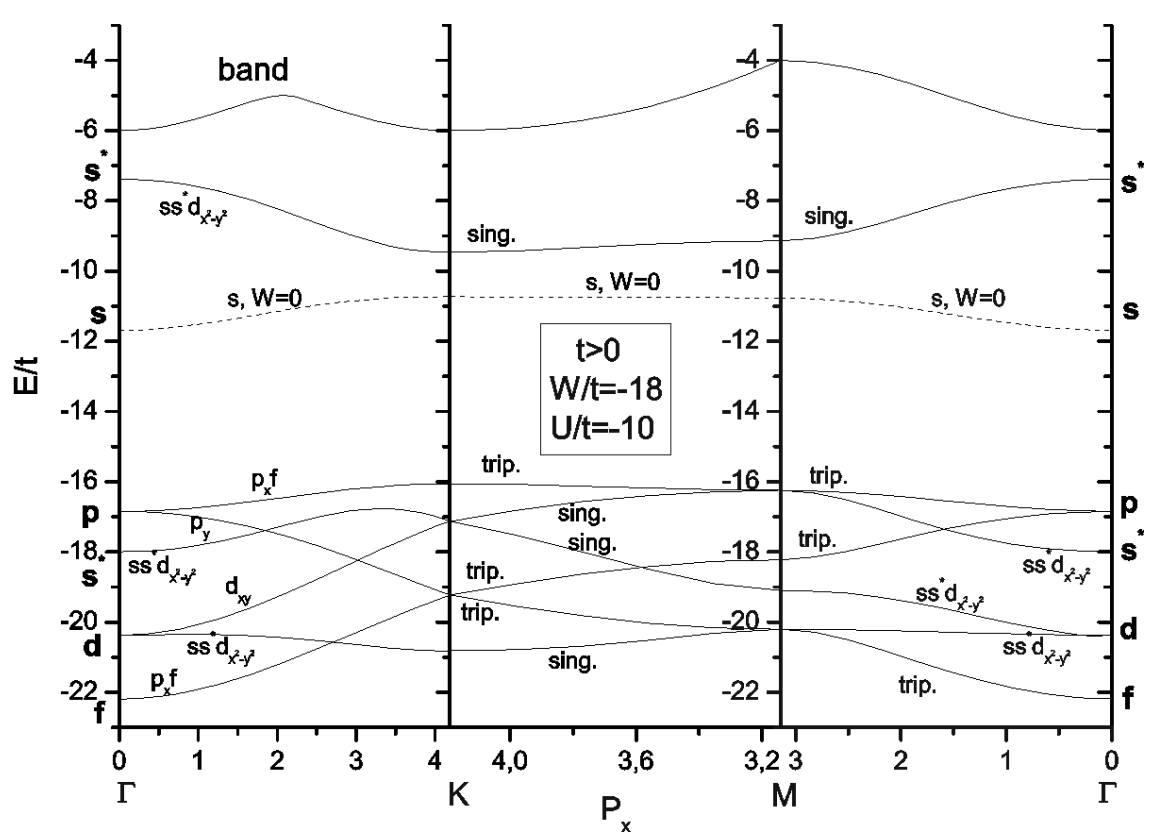

Fig. 5. Dispersion curves across the B.z. for $t>0, W / t=-18$ and $U / t=-10$. Label "trip." means one of the triplet solutions of the coupled $p_{x}+p_{y}+f$-wave pairings; "sing." denotes one of three or four solutions of the four coupled singlet equations for the $s+s^{*}+d_{x^{2}-y^{2}}+d_{x y}$-wave pairing.

The asymptotes are for $W / t=-4$ and $U / t=-6$, so the values of the parameters $U / t=-10, W / t=-18$ put us in the region of the parameter space, where all the solutions exist.

The order of pairing is for $t>0$ reversed: the ground state is $f$-wave, while on $K-M$ line its role is taken by the singlet state. The order $f, d, s^{*}, p, s^{*}$ (from the most to the least bound) is not a precise reversal of the $t<0$ case, as we have additional $s^{*}$-wave solution of intermediate energy. The pure $s$-state is not the weakest bound state for a given $W$, this role is taken by $s^{*}$-wave solution. Let us note a complete change of the shape of the band bottom with the change of the sign of the hopping integral. This is the effect of the hexagonal lattice being non-bipartite.

In conclusion, the properties of the bound states on the hexagonal lattice were calculated for both signs of the n.n. hopping integral. All possible pairing symmetries were examined, binding energies, critical values and dispersion curves across the B.z. were evaluated. The largest stability of $s^{*}$-wave pairs was found for $t<0$ around the band minimum and stabilization of $f$-wave pairing for $t>0$. The conditions and critical values for the existence of various number of $s^{*}$-wave pairing solutions were found and the phase diagram concerning this problem was calculated. 


\section{Acknowledgments}

Support from the Foundation for Polish Science (FNP) is acknowledged. This work was also supported by the State Committee for Scientific Research, project No. 1 P03B 08426.

\section{References}

[1] M. Randeria, J.-M. Duan, L.-Y. Shieh, Phys. Rev. Lett. 62, 981 (1989).

[2] K. Takada, H. Sakurai, E. Takayama-Muromachi, F. Izumi, R.A. Dilanian, T. Sasaki, Nature (London) 422, 53 (2003).

[3] R. Micnas, J. Ranninger, S. Robaszkiewicz, Rev. Mod. Phys. 62, 113 (1990).

[4] H.Q. Lin, Phys. Rev. B 44, 4674 (1991).

[5] A.G. Petukhov, J. Galan, J. Verges, Phys. Rev. B 46, 6212 (1992).

[6] A.S. Blaer, H.C. Ren, O. Tchernyshyov, Phys. Rev. B 55, 6035 (1997).

[7] M. Bak, R. Micnas, Mol. Phys. Rep. 24, 168 (1999); cond-mat/9909089.

[8] S. Basu, R.J. Gooding, P.W. Leung, Phys. Rev. B 63, 100506 (2001).

[9] P. Kornilovitch, Phys. Rev. B 69, 235110 (2004).

[10] D.C. Mattis, The Theory of Magnetism I, Springer-Verlag, Berlin 1988; Rev. Mod. Phys. 58, 361 (1986).

[11] S. Basu, R.J. Gooding, Phys. Status Solidi B 242, 1431 (2005). 